\title{
A Study on a Multi-Objective Optimization Method Based on Neuro-Response Surface Method (NRSM)
}

\author{
Jae-Chul Lee ${ }^{1}$, Ji-Ho Jeong ${ }^{1}$, Hicham Kharoufi ${ }^{1}$ and Sung-Chul Shin ${ }^{1, a}$ \\ ${ }^{1}$ Department of Naval Architecture and Ocean Engineering, Pusan National University, Jangjeon 2-dong, \\ Geumjeong-gu, Busan, South Korea, 609-717
}

\begin{abstract}
The geometry of systems including the marine engineering problems needs to be optimized in the initial design stage. However, the performance analysis using commercial code is generally time-consuming. To solve this problem, many engineers perform the optimization process using the response surface method (RSM) to predict the system performance, but RSM presents some prediction errors for nonlinear systems. The major objective of this research is to establish an optimal design framework. The framework is composed of three parts: definition of geometry, generation of response surface, and optimization process. To reduce the time for performance analysis and minimize the prediction errors, the response surface is generated using the artificial neural network (ANN) which is considered as NRSM. The optimization process is done for the generated response surface by non-dominated sorting genetic algorithm-II (NSGA-II). Through case study of a derrick structure, we have confirmed the proposed framework applicability. In the future, we will try to apply the constructed framework to multi-objective optimization problems.
\end{abstract}

\section{Introduction}

The optimal design of engineering system is considered as multi-objective optimization problem, and the geometry of engineering system severely affects their performance. For this reason, determining an optimal geometry is one of the challenging problems in the initial design stage. In recent years, system optimization based on the performance using the commercial code is a method that was employed for engineering design problem $[1,2]$.

The essence of optimization design process is the performance analysis/evaluation according to the geometry modification. Generally, the performance analysis of the complex engineering system such as marine, aerospace and machinery systems is time-consuming. To reduce the performance calculation time, many researchers try to predict the performance using approximation models. These approximation models represent the relationship between inputs and outputs. The RSM is used to predict the system performance $[3,4,5]$, but the method produces some errors in highly nonlinear problems $[6,7,8]$.

The optimal design problems of marine system based on its performance involve highly nonlinear components, such as structural, hydrodynamic and vibration performances. Many researchers tried to optimize the hull form using the parametric design method. Zhang et al. [9] researched hull form

\footnotetext{
${ }^{a}$ Corresponding author : scshin@pusan.ac.kr
} 
design using the parametric approach. They employed the non-uniform rational b-spline (NURBS) method to represent the hull form. Gregory et al. [10] tried to optimise the hull form in calm and rough water based on the parametric design method. Lee and Choi [11] tried to optimise the hull form in terms of hydrodynamic performance using the form parametric design method. Park et al. [12] tried to optimise hull form of KSUEZMAX using optimization techniques. The parametric design method for geometry representation is a useful technique for geometry optimization based on performance.

Up to now, many relevant papers about optimization based on system performance have been published. To predict the system performance, Shin [13] employed the neuro-fuzzy algorithm to predict the wake distribution, $\mathrm{Xu}$ [14] predicted the maritime safety using the artificial neural network (ANN), and Lee [15] tried the prediction for added resistance in waves using the genetic programming (GP). To optimize the shape based on performance, Kim [16] developed a framework to optimize the stern form based on CFD. In the shape optimization research, CFD analysis was just a means of checking performance of a design process, and it is very time-consuming. Therefore, optimization becomes difficult/impossible using CFD tools because we need to check the system performances for a large number of alternative design cases. To examine alternative design cases and minimise the performance analysis time, a multi-objective optimal design framework that includes performance prediction and an optimization process is essential.

To create an appropriate approximation model for highly nonlinear engineering system, Lee [17] compares the prediction accuracy of the response surface generated by RSM, the kriging method and ANN method, and the analysis of prediction result shows that the prediction values given by ANN are more accurate than those of the other models. We subsequently propose an optimal design framework comprising two principal phases:

1st Phase: To predict the system performance, we generate the response surface using ANN that is considered as NRSM in the proposed framework.

2nd Phase: We optimize the shape in the generated response surface using NSGA- II.

By means of a case study of constraint optimization problem, the effectiveness of the proposed optimal design methodology is verified in view of structural performances.

\section{Framework for optimum design based on NRSM}

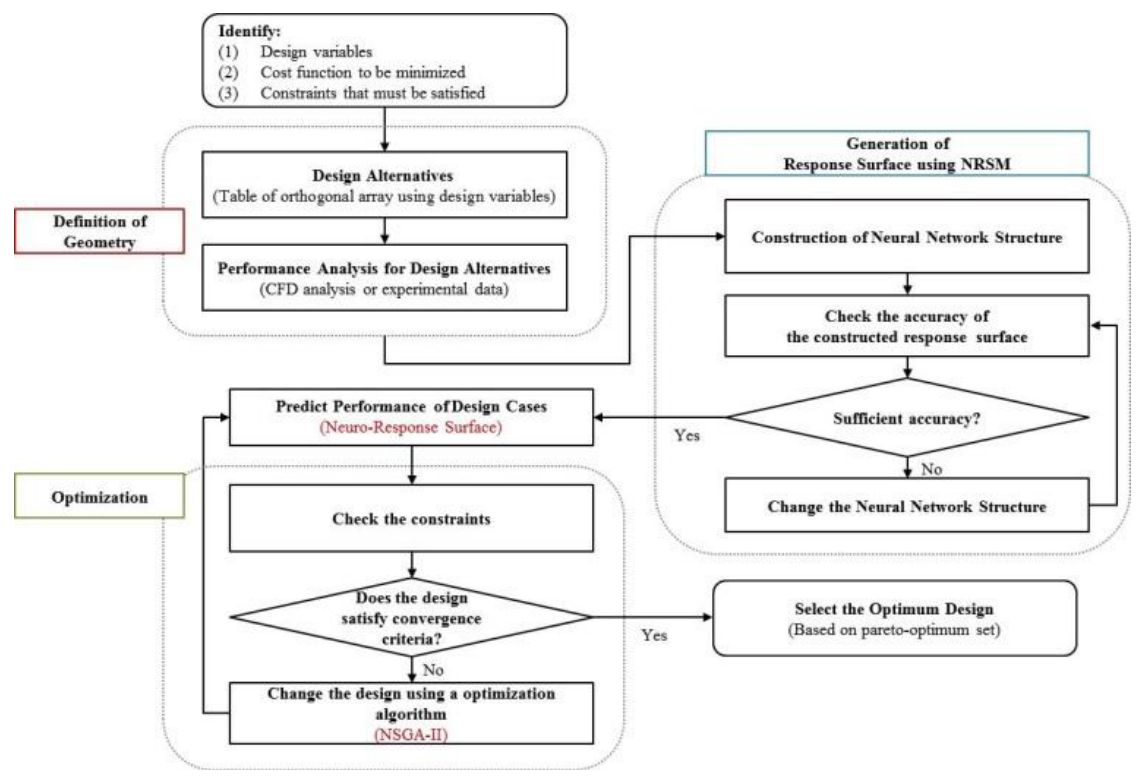

Figure 1. Optimal design framework based on NRSM [17] 
The proposed optimal design framework (Fig. 1) includes three processes:

(1st process: Definition of the geometry)

The framework defines the geometry of the structure using parameterisation. An orthogonal array table [18] is used to systematically generate design alternatives. To check the interaction between the variables and the response, the generated design alternatives are divided into two sets:

- Training data: used to generate the response surface and check the learning accuracy.

- Test data: used to check the prediction accuracy.

\section{(2nd process: Generation of response surface using NRSM)}

The multi-layer perceptron (MLP) is used to generate the response surface. It has three layers: an input layer, a hidden layer, and an output layer. The back-propagation algorithm [19] is used to train the neural network. The prediction accuracy of generated response surface is very important because the optimization process is conducted on it. In order to construct the appropriate response surface, the best structure and the best number of learning cycles for the neural network are prepared and the prediction accuracy of the generated response surface is checked using Test data (1st process). Through this process, we can make appropriate ANN structure for our problem.

After generating the response surface, the performance of various design alternatives can be predicted easily and quickly.

\section{(3rd process: Optimization)}

The optimization process is conducted to generate the response surface. The NSGA-II [20] is used as a multi-objective optimization algorithm.

Finally, the optimum structural design can be selected using the Pareto-optimum set generated by the proposed framework. The framework was constructed using MATLAB.

\section{Case study}

A derrick structure is used for a case study by considering its weight reduction while keeping its safety factor upper than 3 . The accuracy of the framework results has been analysed using ANSYS.

\subsection{Formulation of optimization problem}

The equations below show the formulation process of the optimization problem (Eqs. 1 4):

Find $x_{\mathrm{i}}$

$x_{\mathrm{i}}=$ Design variables $(\mathrm{i}=1,2,3,4,5,6,7)$

to minimize

$f_{1}(x)=$ Steel weight $($ ton $)$

to maximize

$f_{2}(x)=$ Safety factor

Using weighting factor, we can formulate the problem as one function to be minimized:

$$
\mathrm{F}(x)=\left(W_{1} * f_{1}\right)+\left(W_{2} * 1 / f_{2}\right)
$$


where,

$W_{\mathrm{i}}=$ Weighting factor $(\mathrm{i}=1,2)$

Subject to Min $x_{\mathrm{i}} \leq x_{\mathrm{i}} \leq \max x_{\mathrm{i}}(\mathrm{i}=$ number of design variables $)$

$f_{1}(x) \leq$ base design case

$f_{2}(x) \geq 3.0$

Derrick structure consists of 2 beam shapes (I beam and Box beam). Fig. 2 shows the cross section area shape and main dimension.
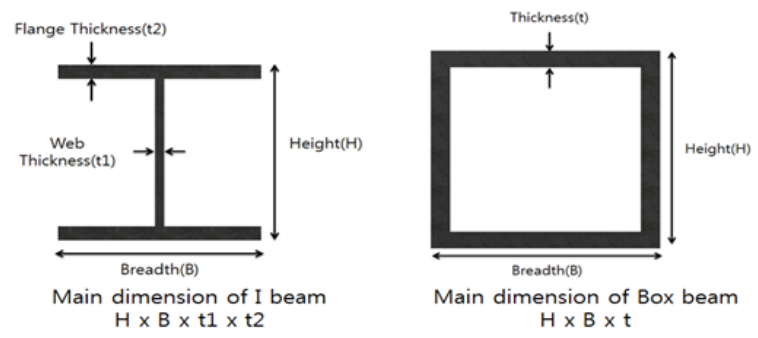

Figure 2. Cross section area shape and main dimension of I beam and Box beam

Seven design variables are considered (Table 1). Fig. 3 ((a) and (b)) shows the design model including the design variables.

Table 1. Design variables.

\begin{tabular}{|c|c|c|}
\hline \multirow{2}{*}{$\begin{array}{c}\text { Breadth } 750 \mathrm{~mm} \\
\text { (I beam) }\end{array}$} & Web thickness & W1 \\
\hline & Flange thickness & F1 \\
\hline \multirow{2}{*}{$\begin{array}{l}\text { Breadth 500mm } \\
\text { (I beam) }\end{array}$} & Web thickness & $\mathrm{W} 2$ \\
\hline & Flange thickness & $\mathrm{F} 2$ \\
\hline \multicolumn{2}{|c|}{ Top Box beam thickness } & $\mathrm{T}$ \\
\hline \multicolumn{2}{|c|}{ Middle Box beam thickness } & M \\
\hline \multicolumn{2}{|c|}{ Bottom Box beam Thickness } & $\mathrm{B}$ \\
\hline
\end{tabular}
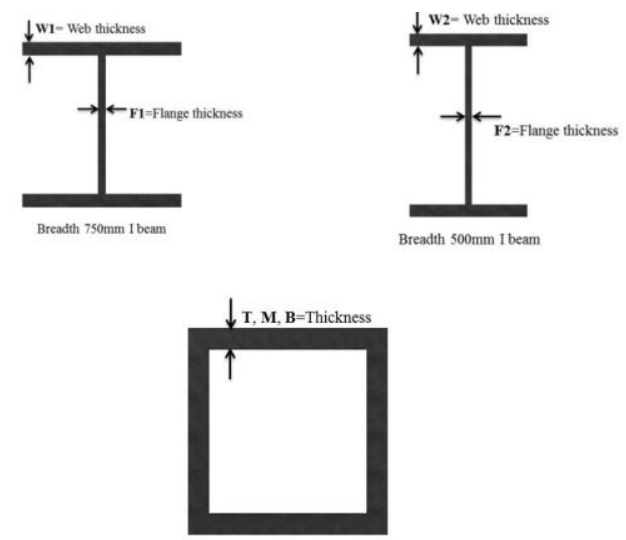

Box beam

(a) Supporting member in derrick (Design model)

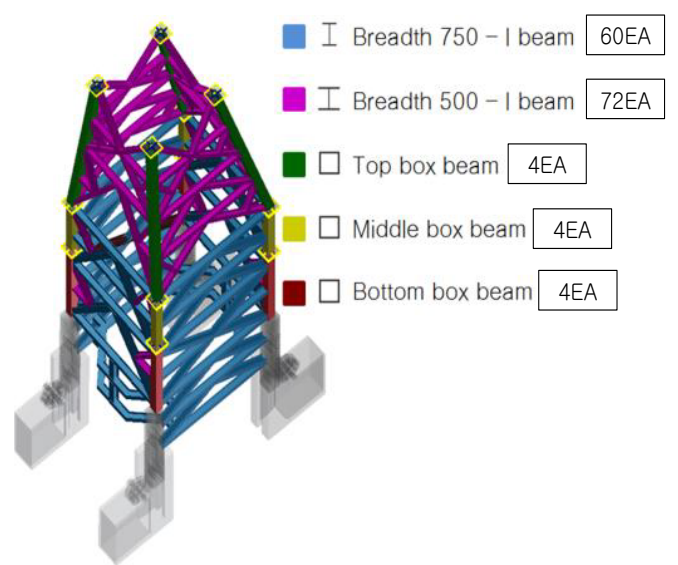

(b) Main dimensions for member (Design variables)

Figure 3. Design model and design variables 


\subsection{Environmental conditions}

Fig. 4 shows the environmental conditions and constraints for calculation. A, B, C, D, E mean the gravity, acceleration of heave motion, rotational velocity of roll and pitch motion, vertical loads (wind pressure) and fixed condition respectively. Fig. 5 and Table 2 show the loading condition.

Table 2. Loading condition.

\begin{tabular}{|c|c|c|c|}
\hline & HC, TDM and etc. & Pipe and etc. & Total \\
\hline Weight & 1,030ton & 600 ton & 1,630 ton \\
\hline Mark (Fig.12) & A, B, C, D & E, F, G & - \\
\hline
\end{tabular}

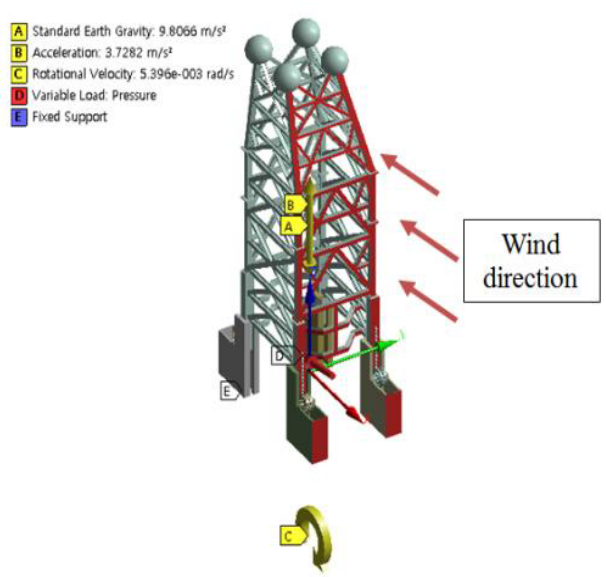

Figure 4. Environmental conditions and constraints

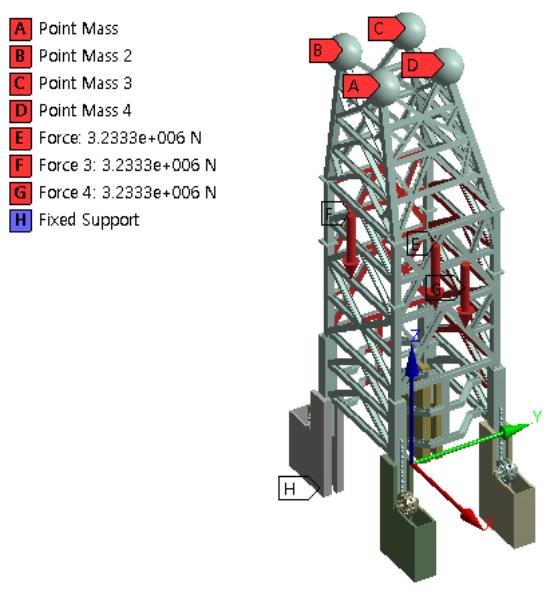

Figure 5. Loading condition

\subsection{Definition of geometry}

The proposed framework defines the shape of the structure by parameterization. The generated geometries are used to generate the response surface. 18 sets of different design alternatives are generated using an orthogonal array table $\left(\mathrm{L}_{18}\left(2^{1} \times 3^{2}\right)\right)$. Table 3 shows design variables and the results of the performance analysis for steel weight and safety factor using the ANSYS. The safety factor is calculated as below:

$$
\text { Safety factor }=\text { Yield stress / Maximum stress }
$$

In equation (5), yield stress is a material property while the maximum stress is calculated on the derrick system.

Design no. 19 is considered as base design case.

Table 3. Design variables and performance analysis results.

\begin{tabular}{|c|c|c|c|c|c|c|c|c|c|}
\hline \multirow{2}{*}{ Case } & \multicolumn{7}{|c|}{ Design variables } & \multicolumn{2}{c|}{ Performance analysis results } \\
\cline { 2 - 10 } & W1 & F1 & W2 & F2 & T & M & B & Steel weight (ton) & Safety factor \\
\hline 1 & 25 & 40 & 20 & 30 & 80 & 80 & 80 & 1279.337 & 2.249 \\
\hline 2 & 25 & 50 & 25 & 40 & 90 & 90 & 90 & 1439.202 & 2.746 \\
\hline 3 & 25 & 60 & 30 & 50 & 100 & 100 & 100 & 1597.343 & 3.203 \\
\hline 4 & 30 & 40 & 20 & 40 & 90 & 100 & 100 & 1390.810 & 2.431 \\
\hline 5 & 30 & 50 & 25 & 50 & 100 & 80 & 80 & 1478.803 & 2.784 \\
\hline 6 & 30 & 60 & 30 & 30 & 80 & 90 & 90 & 1491.352 & 3.126 \\
\hline
\end{tabular}




\begin{tabular}{|c|c|c|c|c|c|c|c|c|c|}
\hline 7 & 35 & 40 & 25 & 30 & 100 & 90 & 100 & 1398.747 & 2.306 \\
\hline 8 & 35 & 50 & 30 & 40 & 80 & 100 & 80 & 1460.352 & 2.728 \\
\hline 9 & 35 & 60 & 20 & 50 & 90 & 80 & 90 & 1560.115 & 3.136 \\
\hline 10 & 25 & 40 & 30 & 50 & 90 & 90 & 80 & 1393.590 & 2.390 \\
\hline 11 & 25 & 50 & 20 & 30 & 100 & 100 & 90 & 1423.310 & 2.756 \\
\hline 12 & 25 & 60 & 25 & 40 & 80 & 80 & 100 & 1498.982 & 3.195 \\
\hline 13 & 30 & 40 & 25 & 50 & 80 & 100 & 90 & 1401.650 & 2.502 \\
\hline 14 & 30 & 50 & 30 & 30 & 90 & 80 & 100 & 1440.041 & 2.717 \\
\hline 15 & 30 & 60 & 20 & 40 & 100 & 90 & 80 & 1526.360 & 3.203 \\
\hline 16 & 35 & 40 & 30 & 40 & 100 & 80 & 90 & 1420.795 & 2.328 \\
\hline 17 & 35 & 50 & 20 & 50 & 80 & 90 & 100 & 1486.953 & 2.823 \\
\hline 18 & 35 & 60 & 25 & 30 & 90 & 100 & 80 & 1511.466 & 3.174 \\
\hline 19 & 35 & 60 & 30 & 50 & 100 & 100 & 100 & 1630.613 & 3.536 \\
\hline
\end{tabular}

After performance calculation of the generated design cases, the response surface was generated using NRSM. Then, we predicted the performance of the design cases in a continuous response surface that was not directly computed. We used 15 sets of data to generate the response surface (Case $1,2,3,5,6,7,9,10,11,13,14,15,17,18$ and 19) and 4 sets of data to check its accuracy (Case 4, 8, 12 and 16). To increase the learning rate for a neural network, all data was used as a normalized value between 0.5 and 1 .

\subsection{Generation of response surface using NRSM}

The number of hidden layers was changed from 1 to 10. Using six hidden layers gave a better result. Therefore, the final structure and the number of learning cycles are 7-6-2 and 6400 respectively. Fig. 6 shows the error convergence in the learning process of the neural network. The error convergence (0.0004) occurs at approximately 6238 iterations.

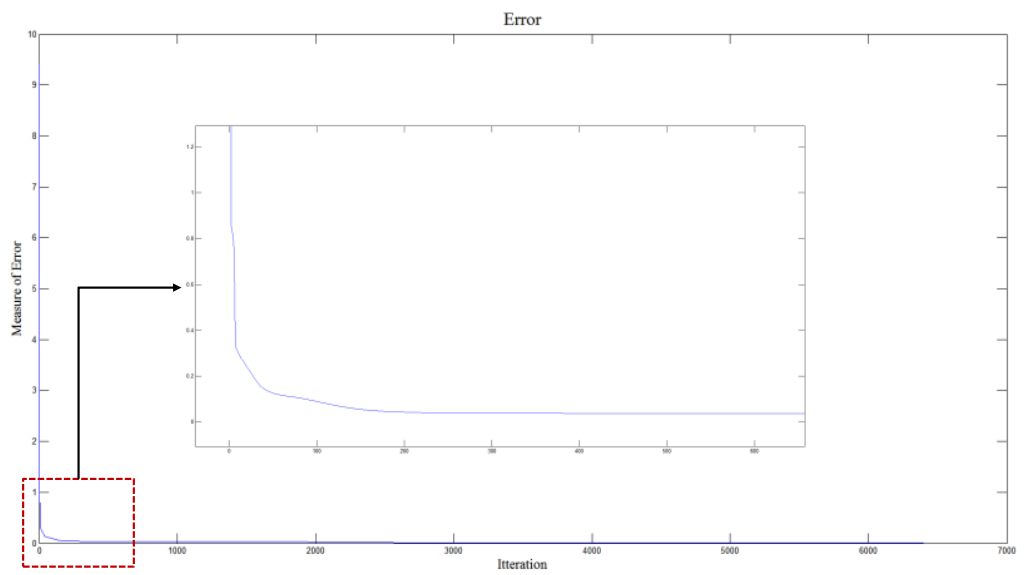

Figure 6. Measure of error

The error is defined in equations (6) and (7). The error value between the output of the network $\left(\mathrm{d}_{\mathrm{j}}\right)$ and the actual value $\left(\mathrm{y}_{\mathrm{j}}\right)$ is used. $\mathrm{L}$ is the number of output neurons.

$$
\begin{aligned}
& \mathrm{e}_{\mathrm{i}}(\mathrm{n})=\mathrm{d}_{\mathrm{j}}(\mathrm{n})-\mathrm{y}_{\mathrm{j}}(\mathrm{n}) \\
& \mathrm{E}(\mathrm{n})=1 / 2 \sum_{\mathrm{j}=1}^{\mathrm{L}} \mathrm{e}_{\mathrm{j}}^{2}(\mathrm{n})
\end{aligned}
$$

Table 4 shows the accuracy of the generated response surface using Training data set. 
Table 4. Analysis results for training data set.

\begin{tabular}{|c|c|c|c|c|}
\hline \multirow{2}{*}{ Case } & \multicolumn{2}{|c|}{ Desired values (ANSYS) } & \multicolumn{2}{c|}{ Prediction values (NRSM) } \\
\cline { 2 - 5 } & Steel weight & Safety factor & Steel weight & Safety factor \\
\hline 1 & 0.500 & 1.000 & 0.500 & 0.992 \\
\hline 2 & 0.728 & 0.752 & 0.720 & 0.746 \\
\hline 3 & 0.953 & 0.591 & 0.962 & 0.587 \\
\hline 5 & 0.784 & 0.736 & 0.782 & 0.728 \\
\hline 6 & 0.802 & 0.615 & 0.799 & 0.607 \\
\hline 7 & 0.670 & 0.966 & 0.665 & 0.965 \\
\hline 9 & 0.900 & 0.612 & 0.893 & 0.612 \\
\hline 10 & 0.663 & 0.919 & 0.656 & 0.919 \\
\hline 11 & 0.705 & 0.747 & 0.698 & 0.751 \\
\hline 13 & 0.674 & 0.861 & 0.666 & 0.868 \\
\hline 14 & 0.729 & 0.764 & 0.724 & 0.771 \\
\hline 15 & 0.852 & 0.591 & 0.850 & 0.601 \\
\hline 17 & 0.796 & 0.721 & 0.797 & 0.724 \\
\hline 18 & 0.830 & 0.600 & 0.833 & 0.604 \\
\hline 19 & 1.000 & 0.500 & 0.979 & 0.501 \\
\hline
\end{tabular}

The structure of the neural network is appropriate, because all of the error values (Error $=$ [(Desired value - Prediction values) / Desired values] $\times 100)$ are below 2.1\% (Case 19). Table 5 and Table 6 show the prediction accuracy of the generated response surface and errors for the test data. Analysis of the results in Table 6 shows that there are still prediction errors. However, to determine the performance in a limited time, the NRSM can give reasonable results for the initial design stage.

Table 5. Analysis results for test data set.

\begin{tabular}{|c|c|c|c|c|}
\hline \multirow{2}{*}{ Case } & \multicolumn{2}{|c|}{ Desired values (ANSYS) } & \multicolumn{2}{c|}{ Prediction values (NRSM) } \\
\cline { 2 - 5 } & Steel weight & Safety factor & Steel weight & Safety factor \\
\hline 4 & 0.659 & 0.897 & 0.658 & 0.924 \\
\hline 8 & 0.758 & 0.759 & 0.751 & 0.721 \\
\hline 12 & 0.813 & 0.593 & 0.806 & 0.616 \\
\hline 16 & 0.701 & 0.953 & 0.688 & 0.930 \\
\hline
\end{tabular}

Table 6. Error of test data set.

\begin{tabular}{|c|c|c|}
\hline \multirow{2}{*}{ Case } & Error (\%) $=[($ Desired value - Prediction values) $/$ Desired values] $\times \mathbf{1 0 0}$ \\
\cline { 2 - 3 } & Steel weight (ton) & Safety factor \\
\hline 4 & $0.2 \%$ & $3.0 \%$ \\
\hline 8 & $0.8 \%$ & $5.0 \%$ \\
\hline 12 & $0.8 \%$ & $3.8 \%$ \\
\hline 16 & $1.9 \%$ & $2.4 \%$ \\
\hline
\end{tabular}

\subsection{Optimization}

Table 7 and Fig. 7 show the parameters for NSGA-II and the pareto-optimum sets as the final result of the framework. To select the final optimum design among the pareto-optimum set, we used a weighting factor. 0.5 is the weighting factors of both Steel weight and Safety factor. The black point is the selected optimum design (Fig. 7).

Table 7. NSGA-II Parameter.

\begin{tabular}{|c|c|}
\hline Parameter & Value \\
\hline Population size & 100 \\
\hline Generation & 1000 \\
\hline Crossover & $30 \%$ \\
\hline Mutation & $2 \%$ \\
\hline
\end{tabular}




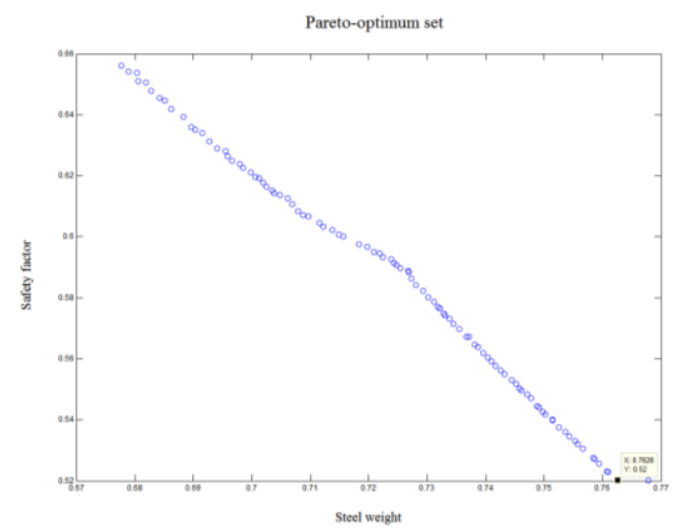

Figure 7. Pareto-optimum set

\subsection{Analysis of Optimum Design}

Table 8 shows the performance analysis results of the constructed framework and ANSYS result. When using the constructed framework, the prediction error is about $10 \%$ (Safety factor), as shown in Table 9.

Table 8. Result analysis.

\begin{tabular}{|c|c|c|c|c|c|c|}
\hline \multicolumn{7}{|c|}{ Design variables for optimum design case } \\
\hline W1 (mm) & W1 (mm) & W1 (mm) & W1 (mm) & W1 (mm) & W1 (mm) & W1 (mm) \\
\hline 25.000 & 25.000 & 25.000 & 25.000 & 25.000 & 25.000 & 25.000 \\
\hline
\end{tabular}

\begin{tabular}{|c|c|c|c|}
\hline \multicolumn{2}{|c|}{ NRSM Framework } & \multicolumn{2}{c|}{ ANSYS Calculation } \\
\hline Steel weight (ton) & Safety factor & Steel weight (ton) & Safety factor \\
\hline 1463.848 & 3.457 & 1472.944 & 3.114 \\
\hline
\end{tabular}

Table 9. Prediction error.

\begin{tabular}{|c|c|}
\hline \multicolumn{2}{|c|}{ Prediction Error (\%) = [(ANSYS Calculation - NRSM Framework) $/$ ANSYS Calculation] $\times \mathbf{1 0 0}$} \\
\hline Steel weight (ton) & Safety factor \\
\hline $0.6 \%$ & $10 \%$ \\
\hline
\end{tabular}

Fig. 8 shows the result of structure analysis. The maximum stress is $1.1238 \mathrm{e} 2 \mathrm{MPa}$.

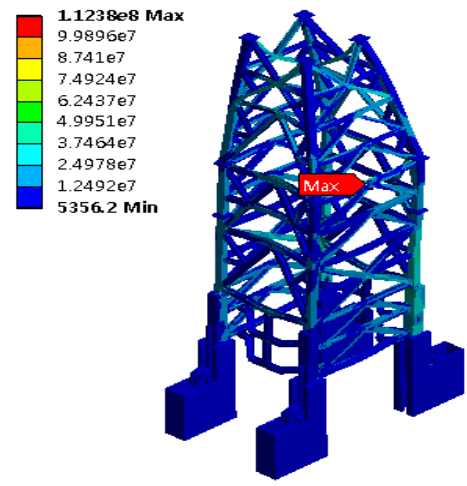

Figure 8. Structure analysis of optimum design 
Finally, the improvement in standards of performance evaluation was analysed, as shown in Table 10 , where all criteria for the optimum design case are satisfied such as steel weight that decreased about $10 \%$ comparing with base design case and safety factor that increased about $3.8 \%$ comparing with the required safety factor.

Table 10. Improvement of performance evaluation criteria.

\begin{tabular}{|c|c|}
\hline Improvement (\%) $=[$ (Base model - Optimization model) $/$ Base model $] \times \mathbf{1 0 0}$ \\
\hline Steel weight (ton) & Safety factor (upper than 3.0) \\
\hline $10 \%$ (Decrease) & $3.8 \%$ (Increase) \\
\hline
\end{tabular}

\subsection{Discussion}

The effectiveness of the proposed framework through multi-objective optimization problem was confirmed by a derrick weight minimization while considering a given safety factor: more than 3 .

In the initial design stage, the shape of various design alternatives considering their performances can be checked in a reduced analysis time using the proposed framework. We considered that optimal design framework presents the starting design point and save the time for optimal design using existing experimental or calculation data based on commercial codes in the initial design stage. However, the proposed framework shows some errors for extrapolation design problems. To increase the prediction accuracy of extrapolation design alternatives, we can add some experimental or calculation data using commercial codes into pre-existing data, or set the range of design variables including all possible design alternatives in the proposed framework (1st process).

\section{Conclusion}

The following conclusions can be drawn from this study:

I. We proposed an optimization framework based on the NRSM:

- The method generates the response surface using the ANN that is considered as NRSM in the proposed framework.

- The system is optimised using the NSGA-II.

II. Through case study on the optimal structure of a derrick, we confirmed the feasibility of the proposed framework for multi-objective optimization problems:

- Selected optimal design case satisfies the design constraints.

III. The proposed framework is considered as a useful engineering system optimization design tool in the initial design stage:

- Save the performance analysis time in the optimization process;

- Widely check the alternative design cases.

Evaluations of the appropriate approximation model for each problem and their application to various optimization problems considering actual design constraints will be conducted in future work.

\section{Acknowledgement}

This work was supported by the National Research Foundation of Korea (NRF) grant funded by the Korea government (MEST) through GCRC-SOP (No. 2011-0030671).

\section{References}

1. D. H. Ko, D. C. Ko, H. J. Lim, J. M. Lee, and B. M. Kim, I. J. P. E. M, 14 (8): pp 1301-1309, (2013)

2. S. P. Jung, Y. G. Kim, and T. W. Park, I. J. P. E. M, 13 (1): pp 57-63, (2012) 
3. K.J. Hong, K. K. Jeon, Y. S. Cho, D. H. Choi, and S. J. Lee (2000), Trans. Korea Soc. Mech. Eng. A, 24(6): 1408-1418, (2000)

4. R. H. Mayers and D. C. Montgomery, 'Response Surface Methodology - Process and Product Optimization Using Designed Experiments', John Wiley \& Sons, (1995)

5. Z. Z. Li, T. H. Cheng, D. J. Xuan, M. Ren, G.Y. Shen, and Y. D. Shen, I. J. P. E. M., 13(9):1641$1645,(2012)$

6. M. Sankaya and A. Gullu, Journal of Cleaner Production, 65: 604-616, (2014)

7. J.M. Salman, Arabian Journal of Chemistry, 7(1): 101-106, (2014)

8. F. Kahraman, Materials and Technology, 43(5): 267-270, (2009)

9. P. Zhang, Journal of Hydrodynamics, 20(6) : pp. 804-810, (2008)

10. G. J. Grigoropoulos, D. S. Chalkias, Computer-Aided Design, 42 : pp. 977-984, (2010)

11. Y.S. Lee, Y.B. Choi, J. N. A. O. E., 46(6): 562-568, (2009)

12. J. H. Park, J. E. Choi and H. H. Chun, Int. J. Nav. Archit. Ocean Eng, 7 : pp. 100-114, (2015)

13. S.C. Shin, Int. J. F. L. I. S., 17(2) : pp.154-159, (2007)

14. J. H. Xu, Information and Management Engineering Communications in Computer and Information Science, 236: 34-38, (2011)

15. J. C. Lee, J. H. Jeong, and S. C. Shin, Proceedings of the Annual Autumn Conference, S. N. A. K. : pp.482-490, (2014)

16. H.J. Kim, H.H. Chun, and H.J. Choi, J. S. N. A. K., 44(6): 564-571, (2007)

17. J. C. LEE, 'Application of Multi-objective Optimization for Marine Systems using NRSM', Ph. D. thesis, Pusan National University, Busan, S. Korea, (2015)

18. P. J. Ross, 'Taguchi Techniques for Quality Engineering' 2nd, McGraw-Hill, (1996)

19. H.N. Robert, IJCNN, 1: pp. $593-605$, (1989)

20. K. Deb, IEEE TRAN. E. C., 6 (2): pp. 182-197, (2002) 